\title{
SLMP53-1 interacts with wild-type and mutant p53 DNA-binding domain and reactivates multiple hotspot mutations
}

\author{
Ana Sara Gomes ${ }^{\mathrm{a}, 1}$, Helena Ramos ${ }^{\mathrm{a}, 1}$, Sara Gomes ${ }^{\mathrm{a}}$, Joana B. Loureiro ${ }^{\mathrm{a}}$, Joana Soares ${ }^{\mathrm{a}}$, \\ Valentina Barcherini $^{\mathrm{a}}$, Paola Monti ${ }^{\mathrm{c}}$, Gilberto Fronza ${ }^{\mathrm{c}}$, Carla Oliveira $^{\mathrm{d}}$, Lucília Domingues $^{\mathrm{d}}$, \\ Margarida Bastos $^{\mathrm{e}}$, Daniel F.A.R. Dourado ${ }^{\mathrm{f}, \mathrm{i}}$, Ana Luísa Carvalho ${ }^{\mathrm{g}}$, Maria João Romãog, \\ Benedita Pinheiro $^{g}$, Filipa Marcelo ${ }^{g}$, Alexandra Carvalho ${ }^{\mathrm{h}}$, Maria M.M. Santos ${ }^{\mathrm{b}}$, Lucília Saraiva ${ }^{\mathrm{a}, *}$ \\ ${ }^{a}$ LAQV/REQUIMTE, Laboratório de Microbiologia, Departamento de Ciências Biológicas, Faculdade de Farmácia, Universidade do Porto, 4050-313 Porto, Portugal \\ ${ }^{\mathrm{b}}$ Research Institute for Medicines (iMed.ULisboa), Faculdade de Farmácia, Universidade de Lisboa, Lisboa, Portugal \\ ${ }^{\mathrm{c}}$ Mutagenesis and Cancer Prevention Unit, IRCCS Ospedale Policlinico San Martino, 16132 Genoa, Italy \\ ${ }^{\mathrm{d}}$ CEB-Centre of Biological Engineering, University of Minho, Campus de Gualtar, 4710-057 Braga, Portugal

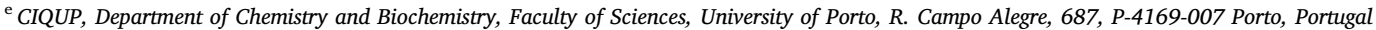 \\ ${ }^{\mathrm{f}}$ School of Chemistry and Chemical Engineering, Queen's University Belfast, UK \\ ${ }^{\mathrm{g}}$ UCIBIO, Departamento de Química, Faculdade de Ciências e Tecnologia, Universidade Nova de Lisboa, 2829-516 Caparica, Portugal \\ ${ }^{\mathrm{h}}$ CNC-Center for Neuroscience and Cell Biology, University of Coimbra, 3004-504 Coimbra, Portugal \\ ${ }^{\mathrm{i}}$ Almac Sciences, Department of Biocatalysis and Isotope Chemistry, Almac House, 20 Seagoe Industrial Estate, Craigavon BT63 5QD, UK
}

\section{A R T I C L E I N F O}

\section{Keywords:}

p53

Mutant

Cancer

Chemotherapy

Reactivator

\begin{abstract}
A B S T R A C T
Background: Half of human cancers harbour TP53 mutations that render p53 inactive as a tumor suppressor. As such, reactivation of mutant (mut)p53 through restoration of wild-type (wt)-like function represents one of the most promising therapeutic strategies in cancer treatment. Recently, we have reported the $(S)$-tryptophanolderived oxazoloisoindolinone SLMP53-1 as a new reactivator of wt and mutp53 R280K with in vitro and in vivo p53-dependent antitumor activity. The present work aimed a mechanistic elucidation of mutp53 reactivation by SLMP53-1.

Methods and results: By cellular thermal shift assay (CETSA), it is shown that SLMP53-1 induces wt and mutp53 R280K thermal stabilization, which is indicative of intermolecular interactions with these proteins. Accordingly, in silico studies of wt and mutp53 R280K DNA-binding domain with SLMP53-1 unveiled that the compound binds at the interface of the p53 homodimer with the DNA minor groove. Additionally, using yeast and p53-null tumor cells ectopically expressing distinct highly prevalent mutp53, the ability of SLMP53-1 to reactivate multiple mutp53 is evidenced.

Conclusions: SLMP53-1 is a p53-activating agent with the ability to directly target wt and a set of hotspot mutp53.

General Significance: This work reinforces the encouraging application of SLMP53-1 in the personalized treatment of cancer patients harboring distinct p53 status.
\end{abstract}

\section{Introduction}

The tumor suppressor protein p53 coordinates a myriad of essential cellular processes, including DNA repair, cell cycle arrest, and death, mostly by acting as a transcription factor [1]. The impairment or loss of p53 is a pivotal event in cancer formation and progression [1]. In about half of human cancers, p53 is inactivated by mutation, with colorectal, head and neck, esophagus, female genital organs, pancreas and skin cancers exhibiting the highest prevalence of TP53 mutations [2,3]. The majority of TP53 mutations are missense and occur within the p53 DNA-binding domain (DBD). Despite the huge diversity of mutations in the TP53 gene, there are six hotspot amino acid residues (R175, G245, $\mathrm{R} 248$, R249, R273, and R282) located in (or close to) the DNA-binding surface with high clinical relevance [2,3]. Mutant p53 (mutp53)

\footnotetext{
Abbreviations: DBD, DNA-binding domain; mutp53, mutant p53; wt, wild-type; CETSA, cellular thermal shift assay; SRB, sulforhodamine B

* Corresponding author.

E-mail address: lucilia.saraiva@ff.up.pt (L. Saraiva).

${ }^{1}$ These authors contributed equally to the work.
} 
proteins have been broadly classified as contact (e.g., R248Q, R273H, and R280K) or structural (e.g., R175H, Y220C, and R282W). In contact mutp53, the amino acid substitution occurs on a residue directly involved in DNA binding with complete or partial loss of p53 transcriptional activity, despite the preservation of wild-type (wt)-like conformation. Conversely, in structural mutp53, localized structural distortions induced by the replacement of amino acid residues lead to p53 thermal destabilization with alteration of the active conformation, and to the subsequent disruption of its normal function [4]. Nevertheless, this classification lacks clinical relevance. In fact, besides p53 loss-of-function, mutp53 can exert a dominant-negative effect over wtp53, and acquire new oncogenic properties, called gain-of-function (GOF) [5]. All these functions of mutp53 have a different impact in tumor cell survival and proliferation, with worst prognosis for GOF mutants [5]. However, it remains unclear why both contact and structural mutants can display these diverse biological functions.

In this way, mutp53 has represented one of the most important candidate targets against cancer. Indeed, many studies have been focused on the development of small-molecule reactivators able to restore wt-like function to mutp53 [5]. Although several small-molecule mutp53 reactivators have been reported to date, only two (PRIMA- $1^{\text {MET }}$ and COTI-2) are under clinical trials, and so far, none has reached the clinic [1,6-8]. As such, several issues remain unclear regarding these mutp53 reactivators, particularly their clinical efficacy in cancer treatment and potential complications of long-term treatments [1,6-8].

Recently, we have reported the (S)-tryptophanol-derived oxazoloisoindolinone, SLMP53-1, as a new reactivator of contact mutp53 R280K, which is also able to activate wtp53 [9,10]. SLMP53-1 restored DNA-binding ability, and subsequent transcriptional activity, to mutp53 R280K. Additionally, it displayed in vitro and in vivo p53-dependent antitumor activity against tumors bearing wtp53 or mutp53 R280K, with no apparent undesirable toxicity [10]. However, there was still a lack of understanding about the molecular mechanism by which SLMP53-1 reactivates mutp53 R280K, as well as its potential to reactivate other mutp53 besides R280K. An additional aspect requiring further elucidation was related to the observed biological inactivity of SLMP53-1 (R)-enantiomer [10].

In the present work, it is shown that SLMP53-1 binds to wt and mutp53 R280K, and also reactivates a set of hotspot mutp53.

\section{Material and methods}

\subsection{Compounds}

The (S)-tryptophanol-derived oxazoloisoindolinone SLMP53-1 was synthesized as described [10]. For all experiments, SLMP53-1 was dissolved in dimethyl sulfoxide (DMSO) from Sigma-Aldrich (Sintra, Portugal).

\subsection{Construction of yeast and mammalian expression vectors}

\subsubsection{Yeast expression vectors}

The yeast expression vectors pLLS89 (GAL1-10, LEU2) encoding full-length human mutp53 (R175H, G245D, G245S, R248Q, R248W, R273H, R273C or R282W) were constructed through SgraI/StuI (New England Biolabs, Ipswich, Massachusetts, USA) digestion of available pLS76 (ADH1, LEU2) vectors [11] and subsequent ligation (T4 DNA ligase, New England Biolabs) of the gel purified mutp53 fragment in a SgraI/StuI digested and gel purified pLLS89-based vector (generous gift of Prof. Alberto Inga). The creation of the mutp53 expression vectors was confirmed after XL1-Blue E. coli extraction (GenEluteTM Plasmid Miniprep Kit, Sigma-Aldrich, St. Louis, Missouri, USA) by digestion and DNA sequencing (BMR Genomics, Padua, Italy).

\subsubsection{Mammalian expression vectors}

The full-length coding sequence of mutp53 was PCR-amplified from the yeast vector with Vent DNA polymerase (New England Biolabs, Werfen, Porto, Portugal), using the primers pair 5' GGG GTA CCA TGG AGG AGC CGC AGT CAG $3^{\prime}$ and $5^{\prime}$ CCG CTC GAG TCA GTC TGA GTC AGG CCC TTC $3^{\prime}$, where the restriction sites for KpnI/XhoI (in bold) were included, respectively. The PCR products and the mammalian expression vector pcDNA3 (Invitrogen, Alfagene, Lisboa, Portugal) were digested with KpnI/XhoI (New England Biolabs, Werfen), purified from agarose gel, and ligated with T4 DNA ligase (Promega, VWR, Carnaxide, Portugal), originating the expression vectors pcDNA3mutp53. These constructs were propagated in NZY5 $\alpha$ E. coli cells (NZYTech, Lisboa, Portugal) as described [12]. The sequence of each mutp53 in the constructed vectors was confirmed by sequencing (Eurofins GATC Biotech, Konstanz, Germany) with specific pcDNA3 primers. pcDNA3-mutp53 vectors were extracted using the PureYieldTM Plasmid Miniprep System kit (Promega, VWR).

\subsection{Modeling}

The starting structure for the modeling was the crystal structure of the wtp53 DBD tetramer bound to a DNA target (Protein Data Bank (PDB) code: 2AC0) [13]. Missing hydrogen atoms were added to these structures according to the assigned protonation states. The DNA bound mutp53 R280K was obtained by replacing the guanidine group in residue 280 by the butylammonium side chain of lysine in the wtp53 model, and was subjected to a geometry optimization with the Amber 18 (parm99SB) force field [14].

The ligands (S)-tryptophanol-derived oxazoloisoindolinone SLMP53-1 and the corresponding enantiomer $(R)$-tryptophanol-derived oxazoloisoindolinone were geometry optimized in Gaussian09 using B3LYP [15] and a Polarizable Continuum Model (PCM) solvent description [16]. Atomic partial charges were calculated resorting to the Restrained Electrostatic Potential (RESP) [17] method from HF/6$31 \mathrm{G}(\mathrm{d})$ single point energy calculations. The zinc atoms in p53 were described with the semi-bonded model $[18,19]$.

\subsubsection{Molecular docking}

The ligands SLMP53-1 and respective enantiomer were docked to the wtp53 and mutp53 R280K DBD models. Molecular docking was performed using the AutoDock 4.2 suite of programs with the Lamarckian genetic algorithm (LGA) [20]. A grid box was centered on residue 280 of chain A. A total of 100 LGA runs were carried out for each DNA:protein complex or free protein. The population was 300 , the maximum number of generations was 27,000 and the maximum number of energy evaluations was 2,500,000.

\subsubsection{Molecular dynamics (MD) simulations}

MD simulations were performed for the following complexes: DNA:wtp53 with SLMP53-1 and its enantiomer; DNA:mutp53 R280K with SLMP53-1 and its enantiomer. All MD simulations were performed using the Amber 18 molecular dynamics program with the parm99SB [14] and GAFF force fields [21]. The structures were placed within an octahedral box of TIP3P waters [22,23] and counter ions were added to make the entire system neutral. The systems were subjected to two initial energy minimizations and to 500 ps of equilibration in a NVT ensemble using Langevin dynamics with small restraints on the protein $(10 \mathrm{kcal} / \mathrm{mol})$ to heat the system from 0 to $300 \mathrm{~K}$. Production simulations were carried out at $300 \mathrm{~K}$ in the NPT ensemble using Langevin dynamics with a collision frequency of $1.0 \mathrm{ps}^{-1}$. Constant pressure periodic boundary conditions were imposed with an average pressure of $1 \mathrm{~atm}$. Isotropic position scaling was used to maintain pressure with a relaxation time of 2 ps. The time step was set to $2 \mathrm{fs}$. SHAKE constraints were applied to all bonds involving hydrogen atoms [24,25]. The particle mesh Ewald (PME) method [26] was used to calculate electrostatic interactions with a cut off distance of $10 \AA$. Three replicas with different initial velocities were performed for each system. The total combined time of the simulations was $280 \mathrm{~ns}$. The reference structures of the 
simulations were calculated as described [27].

\subsection{Yeast assay}

Saccharomyces cerevisiae CG379 (Yeast Genetic Stock Center, University of California, Berkeley, CA, USA) was transformed with pLLS89 expressing human mutp53 (or empty vector as control), using the LiAc/SS Carrier DNA/PEG method [28]. Yeast cells expressing human wtp53 were obtained in previous work [10]. For expression of human wt or mutp53, cells (routinely grown in minimal selective medium) were incubated in galactose selective medium with all the amino acids required for yeast growth $(50 \mu \mathrm{g} / \mathrm{mL})$ except leucine as described [10], in the presence of $10 \mu \mathrm{M}$ of SLMP53-1 or $0.1 \%$ DMSO, for approximately $42 \mathrm{~h}$ (time required by control yeast incubated with DMSO to achieve $0.4 \mathrm{OD}_{600}$ ). Yeast growth was analyzed by colonyforming unit (CFU) counts as described [10]. Percentage of growth inhibition was calculated considering the wtp53-induced yeast growth inhibition as $100 \%$.

\subsection{Human tumor cell lines and growth conditions}

Human colon adenocarcinoma HCT116 cell lines expressing wt p53 were provided by B. Vogelstein (The Johns Hopkins Kimmel Cancer Center, Baltimore, MD, USA); human breast adenocarcinoma MDA-MB231 (expressing mutp53 R280K) and MDA-MB-468 (expressing mutp53 R273H) cells, and human non-small cell lung cancer NCI-H1299 cells, lacking p53 expression due to a homozygous partial deletion of the TP53 gene, were purchased from ATCC (Rockville, Maryland, USA). Cells were cultured in RPMI-1640 with UltraGlutamine (Lonza, VWR), supplemented with $10 \%$ fetal bovine serum (Merck Millipore, VWR), and incubated at $37{ }^{\circ} \mathrm{C}$ with $5 \% \mathrm{CO}_{2}$. Cells were routinely tested for mycoplasma infection using the MycoAlert ${ }^{\mathrm{TM}}$ PLUS mycoplasma detection kit (Lonza, VWR). Cells were recently characterized and authenticated, by short tandem repeat DNA profiling [29].

\subsection{Cellular thermal shift assay (CETSA)}

To evaluate drug target interactions in cells, the CETSA analysis was performed basically as described [30-32]. Briefly, HCT116 or MDAMB-231 cell lysates were incubated with SLMP53-1 or solvent for $1 \mathrm{~h}$ at room temperature, and then heated at different temperatures for $3 \mathrm{~min}$, cooled to room temperature for $3 \mathrm{~min}$, and placed on ice. Soluble protein was detected by western blot. At $39^{\circ} \mathrm{C}$ (HCT116) or $40^{\circ} \mathrm{C}$ (MDAMB-231), the increase in non-denatured p53 of lysates treated with 1-50 $\mu \mathrm{M}$ SLMP53-1 was calculated relative to solvent at $25^{\circ} \mathrm{C}$, set as 1 .

\subsection{Transient transfection assay of mutp53 in human NCI-H1299 tumor} cells

NCI-H1299 cells were transfected at $7.5 \times 10^{3}$ cells/well density in 96-well plates with $75 \mathrm{ng}$ of pcDNA3-mutp53 (or empty vector), using ScreenFect ${ }^{\circledR}$ A (ScreenFectA GmbH, GRiSP, Porto, Portugal) according to the manufacturer's instructions.

\subsection{Sulforhodamine B (SRB) assay}

NCI-H1299 transfected cells were seeded in 96-well plates at $7.5 \times 10^{3}$ cells/well density, followed by analysis of SLMP53-1 effect on cell proliferation after $48 \mathrm{~h}$ treatment with serial dilutions (3.13-50 $\mu \mathrm{M})$ of compound, as described [33]. The solvent (DMSO; maximum concentration used $0.25 \%$ ) was included as control. IC $\mathrm{IC}_{50}$ (concentration that causes $50 \%$ of growth inhibition) values were determined as described [33].

\subsection{Western blot analysis}

To evaluate the expression levels of human wt and mutp53 in yeast, cells were grown in galactose selective medium for $42 \mathrm{~h}$. To evaluate the expression levels of p53 targets (in MDA-MB-468 cells) and ectopic human mutp53 (in transfected NCI-H1299 cells), cells were seeded in 6well plates at $2.25 \times 10^{5}$ cells/well density for $48 \mathrm{~h}$. Yeast and human tumor cells were lysed and protein fractions were analyzed as described [33]. Antibodies are listed in Table S1.

\subsection{Statistical analysis}

Data were statistically analyzed using the GraphPad Prism 7 (GraphPad software, California, USA). Differences between means were tested for significance using the Student's $t$-test $\left({ }^{* *} p<.01\right.$; $* * * p<.001)$.

\section{Results}

\subsection{SLMP53-1 binds to wt and mutp53 R280K}

To uncover the molecular mechanism by which SLMP53-1 reactivates wtp53 and mutp53 R280K, we performed the CETSA analysis. This technique enlightens about the possibility of direct binding of small molecules to macromolecules. Specifically, it gives insights about the ability of small molecules to induce thermal stabilization of cellular proteins as a consequence of intermolecular interactions [34]. As such, using HCT116 and MDA-MB-231 tumor cells expressing wtp53 and mutp53 R280K, respectively, it was evaluated the impact of SLMP53-1 on wt and mutp53 R280K thermal stabilization. For that, the amount of soluble wt and mutp53 R280K was measured upon heating of HCT116 or MDA-MB-231 cell lysates. In fact, whereas unbound (non-stabilized) proteins denature and precipitate after heating, ligand-bound (stabilized) proteins remain in solution [34]. The results showed that $10 \mu \mathrm{M}$ SLMP53-1 induced a slight mutp53 R280K thermal stabilization at $39^{\circ} \mathrm{C}$ and $40^{\circ} \mathrm{C}$ (Fig. 1A). Consistently, at $40^{\circ} \mathrm{C}$, SLMP53-1 caused concentration-dependent mutp53 R280K thermal stabilization, with a two-fold increase of non-denatured mutp53 R280K thermal stabilization achieved at $50 \mu \mathrm{M}$ SLMP53-1, when compared to solvent (Fig. 1B). In fact, considering the amount of mutp53 R280K obtained with DMSO at $25^{\circ} \mathrm{C}$, a complete protein stabilization was achieved with $50 \mu \mathrm{M}$ SLMP53-1 at $40^{\circ} \mathrm{C}$. Similarly, in wtp53-expressing tumor cells, SLMP53-1 induced a dose-dependent thermal stabilization at $39^{\circ} \mathrm{C}$ (Fig. 1C), with a complete stabilization achieved with $10 \mu \mathrm{M}$ (Fig. 1C). These results showed that SLMP53-1 led to wt and mutp53 R280K thermal stabilization, which was indicative of intermolecular interactions between the compound and wt/mutp53. In fact, these results were further supported by microscale thermophoresis (MST) analysis, using recombinant human mutp53 R280K DBD, which unveiled a direct interaction of SLMP53-1 to this contact mutp53 (Kd of $10.7 \mu \mathrm{M}$, Fig. S1).

\subsection{SLMP53-1 potentially binds to a hydrophobic pocket formed at the interface of the p53 homodimer with the DNA minor groove}

To predict SLMP53-1 binding in a more biologically relevant context with p53 DNA recognition, the models of wt and mutp53 R280K DBD tetramer in complex with DNA were used. Consistently with previous data [13], the MD simulations showed that, in the wtp53 DBD crystal structure, the R280 establishes two hydrogen bonds with a conserved guanine base at the DNA major groove (Fig. 2A). Moreover, as reported [12] in mutp53 R280K, the arginine to lysine substitution abolishes these direct interactions with DNA (Fig. 2A), and no substantial differences were found in the structural motifs' conformational rearrangements. Docking and MD simulations of wt and mutp53 R280K DBD with SLMP53-1 showed that the compound binds at the interface of the p53 homodimer with the minor groove of the DNA target half-site 
A

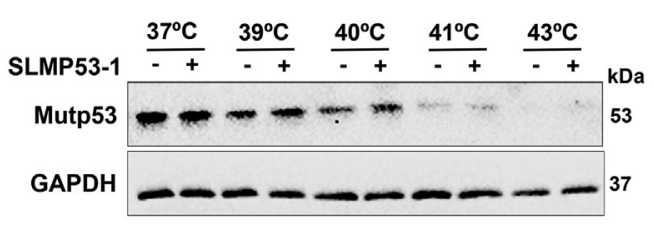

B

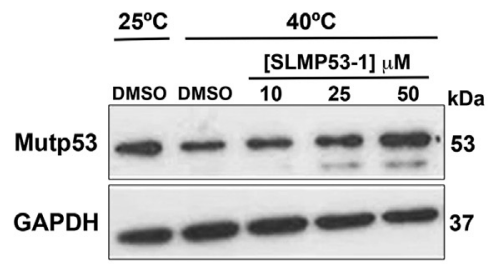

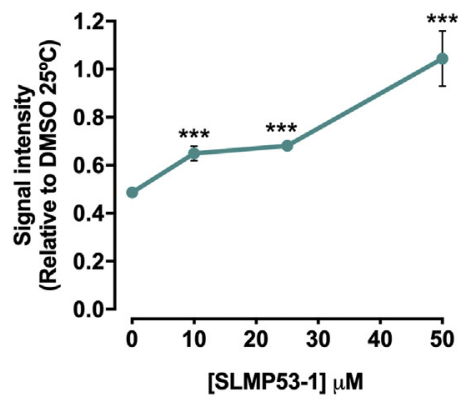

C
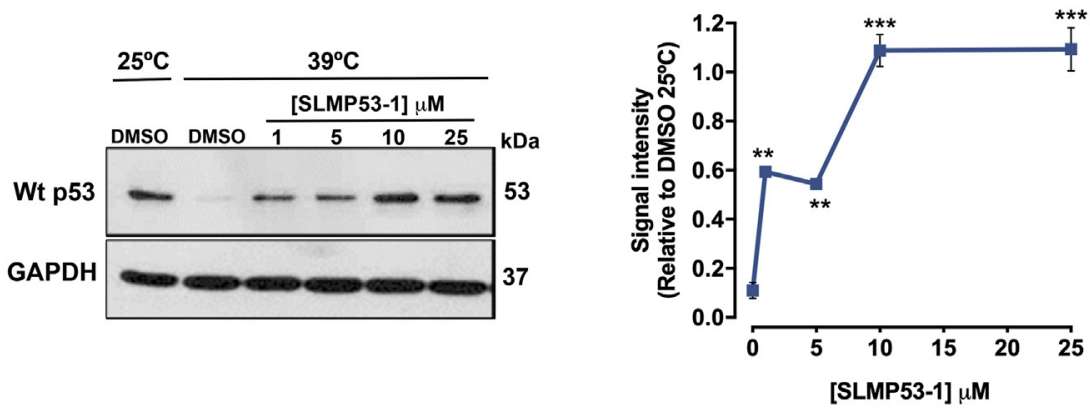

Fig. 1. SLMP53-1 thermally stabilizes wt and mutp53 R280K. CETSA experiments were performed in mutp53 R280K-expressing MDA-MB-231 lysates (A) and (B) and wtp53-expressing HCT116 lysates (C). (A) Lysate samples treated with DMSO ( - ) or 10 $\mu$ M SLMP53-1 (+) were heated at different temperatures; immunoblot represents one of three independent experiments. Lysate samples treated with increasing concentrations of SLMP53-1 were heated at $40{ }^{\circ} \mathrm{C}(\mathrm{B})$ or $39^{\circ} \mathrm{C}(\mathrm{C})$; immunoblot represents one of five independent experiments; plotted data represent the amount of non-denatured mutp53 after heating in SLMP53-1-treated lysates relatively to DMSO at $25{ }^{\circ} \mathrm{C}$ (set as 1); data are mean \pm SEM of five independent experiments; values significantly different from DMSO at $40{ }^{\circ} \mathrm{C}:{ }^{*} p<<.01$, $* * * p<.001$ (unpaired Student's $t$-test). In (A-C), GAPDH was used as a loading control.

(Fig. 2B). This pocket is located on the opposite DNA side from residue 280. SLMP53-1 interacted with p53, mainly with a methionine from each monomer (M243A and M243B), through its indole moiety via sulfur- $\pi$ and methyl- $\pi$ interactions, being closer to M243A (Fig. 2B and C; Table S2). Furthermore, these interactions were closer in mutp53 R280K:SLMP53-1 compared to wtp53:SLMP53-1 complex (Table S2). The isoindolinone moiety of SLMP53-1 further established stacking interactions with the DNA bases (DA18, DT19, DA6, DT7), and a hydrogen bond (with DG12) through its carbonyl group (Fig. 2B and C; Table S2). Consequently, the loss of direct contacts between residue 280 and the DNA major groove was partially compensated by the indirect protein-DNA interactions mediated by SLMP53-1 at the DNA minor groove (Fig. 2B and C).

Although the SLMP53-1 enantiomer could bind to the same pocket in the wtp53 and mutp53 R280K DBD models, its different arrangement prevented the formation of the same interactions established by SLMP53-1 with p53. In fact, due to its spatial orientation, the SLMP53-1 enantiomer interacted mainly with the DNA minor groove in detriment of protein interactions (Fig. 2D), justifying its inactivity as a mutp53 reactivator [10].

\subsection{SLMP53-1 reactivates distinct hotspot mutp53}

It was previously demonstrated that SLMP53-1 was able to restore wt-like function to contact mutp53 R280K, but not to structural mutp53 Y220C [10]. Based on that, we interrogated whether the activity of SLMP53-1 was restricted to mutp53 R280K, or instead it would be also observed in other mutp53, including structural mutp53. To answer this question, the yeast assay, previously developed to screen reactivators of mutp53 R280K and Y220C [10], was extended to other contact (R248Q, R248W, R273H, R273C) and structural (R175H, G245D, G245S, $\mathrm{R} 282 \mathrm{~W})$ mutp53, known for their high prevalence and clinical relevance in human cancers [35-38]. To this end, the heterologous expression of each human mutp53 was carried out in yeast, and their effect on the growth of transformed yeast was analyzed (Fig. 3A and B). By western blot analysis, we confirmed no substantial differences among mutp53 protein expression levels (Fig. 3A). Contrary to the growth inhibitory effect induced by wtp53, none of the expressed mutp53 considerably interfered with yeast cell growth (Fig. 3B, DMSO; Fig. S2). Since potential reactivators of mutp53 would restore the wtlike growth inhibitory effect to mutp53 expressed in yeast, as observed for R280K [10], we next evaluated the effect of SLMP53-1, at $10 \mu \mathrm{M}$ (concentration that caused the maximal effect on mutp53 R280K, reestablishing over 79\% of wtp53-induced growth inhibitory effect) [10], on the growth of mutp53-expressing yeast cells. Despite the negligible effect on G245S and R273C, SLMP53-1 restored the wtp53-induced growth inhibition to the remaining tested mutp53 by $>50 \%$ (Fig. $3 \mathrm{~B}$ ).

To validate the results obtained in yeast, the effect of SLMP53-1 was evaluated on the same set of hotspot mutp53 ectopically expressed in p53-null NCI-H1299 tumor cells, through analysis of cell proliferation by SRB assay, after $48 \mathrm{~h}$ treatment (Fig. 3C and D). No considerable differences among mutp53 protein expression levels were detected by western blot analysis in mutp53-transfected NCI-H1299 cells (Fig. 3C). Notably, like in yeast, SLMP53-1 caused a significant reduction of the $\mathrm{IC}_{50}$ values in NCI-H1299 cells expressing R175H, G245D, R248Q, R248W, R273H, and R282W, when compared to empty vector (Fig. 3D). These results further supported the ability of SLMP53-1 to reactivate distinct contact and structural hotspot mutp53. Moreover, they reinforced the mutp53-dependent cytotoxic effect of SLMP53-1 evidenced in previous work by knocking down mutp53 R280K by siRNA, in MDA-MB-231 tumor cells [10].

To further confirm the growth inhibition induced by SLMP53-1 in mutp53-transfected tumor cells, this effect was also evaluated in human breast adenocarcinoma MDA-MB-468 cells endogenously expressing one of the most prevalent hotspot mutp53 (R273H). As expected, a significant growth arrest was observed (Fig. 3E) with an $\mathrm{IC}_{50}$ value of $10.3 \pm 2.4 \mu \mathrm{M}(n=5)$ determined by SRB assay, after $48 \mathrm{~h}$ treatment with SLMP53-1. This was associated with alterations in the protein 

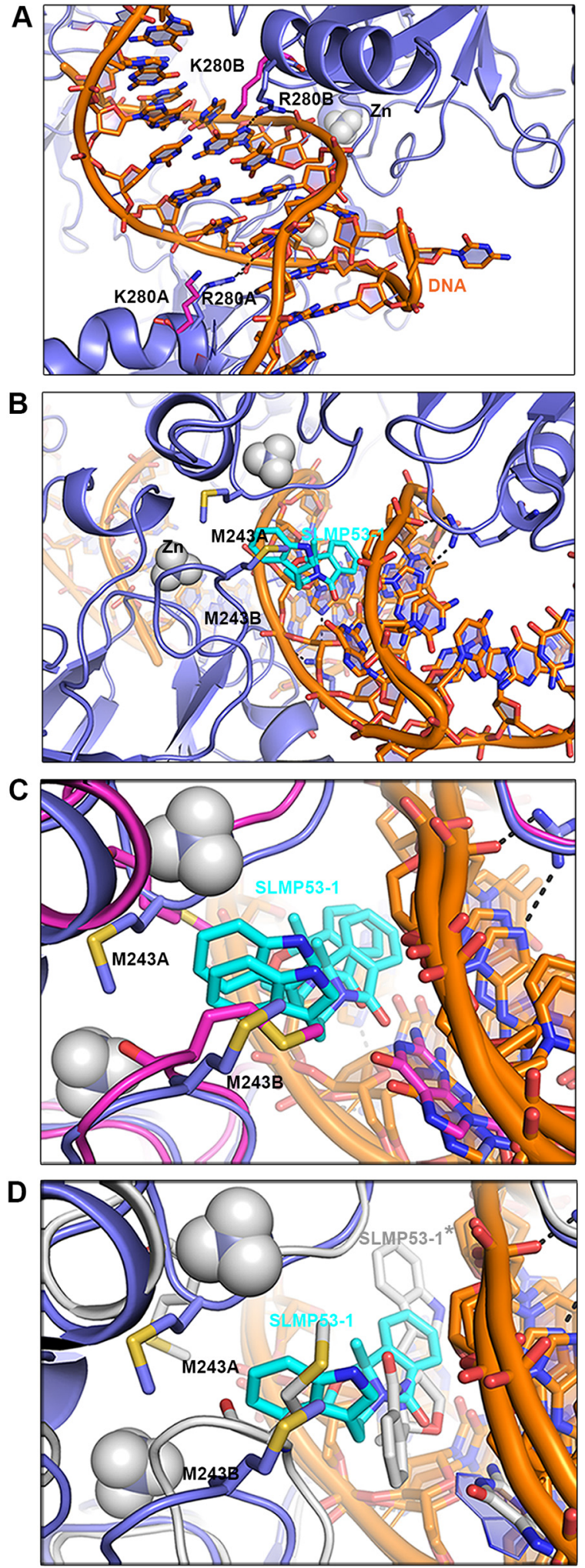

Fig. 2. SLMP53-1 potentially binds at the interface of the p53 homodimer with the DNA minor groove. (A-D) MD reference structures. (A) Image from the DNA major groove side showing the wtp53:SLMP53-1 complex superposed with mutp53 R280K:SLMP53-1 complex (only residue K280, in pink, is depicted from the mutp53 R280K:SLMP53-1 complex). (B) Image from the DNA minor groove side showing the binding of SLMP53-1 to the protein and to the DNA. (C) Superposition of wtp53:SLMP53-1 complex (in purple) with mutp53 R280K:SLMP53-1 complex (in pink) showing the similar binding pose for SLMP53-1. (D) wtp53:SLMP53-1 complex (in purple) superposed with wtp53:SLMP53-1 enantiomer (*) complex (in gray). levels of p53 transcriptional targets. In fact, $10.3 \mu \mathrm{M}$ SLMP53-1 increased the levels of the cell cycle inhibitor p21 and the pro-apoptotic protein PUMA, while decreasing the anti-apoptotic BCL-2 protein levels (Fig. 3F).

\section{Discussion}

The promising pre-clinical results attained with SLMP53-1 in our previous work [10] led us to further delve into the molecular mechanism of action of this potential anticancer drug candidate. The results obtained in this work support a direct interaction of SLMP53-1 to wt and mutp53 with subsequent enhancement of their thermal stability. It is worth noting that despite this thermal stability, additional results unveiled that SLMP53-1 did not interfere with wt/mutp53 half-life (Fig. S3), supporting that its activity is not related to an inhibition of p53 degradation pathways.

To better understand the binding mode of SLMP53-1 to wt and mutp53 R280K, in silico docking studies were performed using structural models of wtp53 tetramer in complex with DNA and the equivalent model with R280K mutagenesis. With this strategy, we intended to simulate a more biologically relevant context, in which the p53 transcriptional activity depends on its DNA-binding ability. In fact, it was previously demonstrated that SLMP53-1 enhanced wtp53 and mutp53 R280K DNA-binding ability, at the promoter regions of target genes, and subsequent transcriptional activity [10]. In the presence of DNA, the $\mathrm{p} 53$ protein organizes as a dimer of dimers (homotetramer) [39]. The results showed that SLMP53-1 binds to a hydrophobic pocket of the p53 homodimer, establishing an interface with the DNA minor groove, and compensating for the loss of direct contacts between the K280 residue and the DNA. Interestingly, the superposition of wt and mutp53 R280K models showed that SLMP53-1 has a similar binding pattern to the wtp53 model, which is consistent with the reported activation of wtp53 by SLMP53-1 [10]. The in silico studies also revealed that the two SLMP53-1 enantiomers presented different binding patterns to p53, therefore justifying the described inability of the SLMP53-1 enantiomer to reactivate both wt and mutp53 R280K [10]. In fact, whereas SLMP53-1 interacts with the protein via sulfur- $\pi$ and sulfur-hydrogen bonds and with DNA via stacking interactions and a hydrogen bond, its enantiomer interacts mainly with the DNA minor groove. As such, SLMP53-1 seems to bridge an extra interaction between the protein and DNA, on the opposite site to the mutation, rescuing the DNA binding and subsequent transcription activity to mutp53.

This work also showed that, besides wt and mutp53 R280K [10], SLMP53-1 also has the ability to reactivate other hotspot p53 mutations with high clinical relevance. The ability of SLMP53-1 to reactivate wtp53, as well as contact and structural mutp53, supports that its molecular mechanism of action is irrespective of the formation of pockets derived from specific mutation sites. In fact, in silico models of wtp53, contact mutp53 R280K, and structural mutp53 R282W (Fig. S4) indicate that the region of L3 (involved in the homodimer interface) is also conserved in this particular structural mutp53.

Altogether, the results herein obtained denote a distinct molecular mechanism of mutp53 reactivation by SLMP53-1, non-dependent on covalent bonds or mutation-created hydrophobic pockets as described for other mutp53 reactivators. In fact, PRIMA- $1^{\mathrm{MET}}$ is transformed into a metabolite that is a Michael acceptor, which binds covalently to p53 free cysteines (C124 and C277), with adducts formation, inducing protein stabilization in both contact and structural mutp53 [40,41]. On the other hand, the carbazole PK08325 and the pyrazole derivative PK708826 bind to a hydrophobic pocket in the cleft induced by the Y220C amino acid substitution in mutp53 structure [42,43]. Since wt and many mutp53 do not have well-defined pockets to accommodate ligands (particularly contact mutants, such as R280K), our work highlights alternatives to the design and development of new mutp53 reactivators. Consistently, it was previously suggested that an interesting strategy to reactivate mutp53 would rely on bridging the interaction 
A

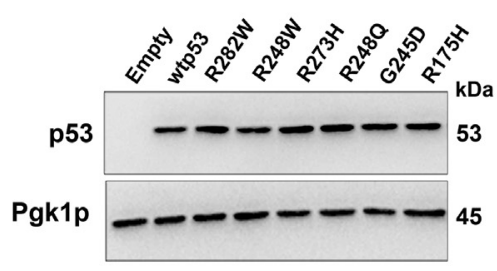

C

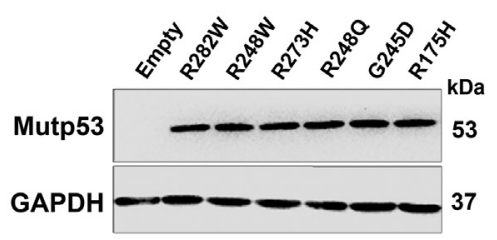

B

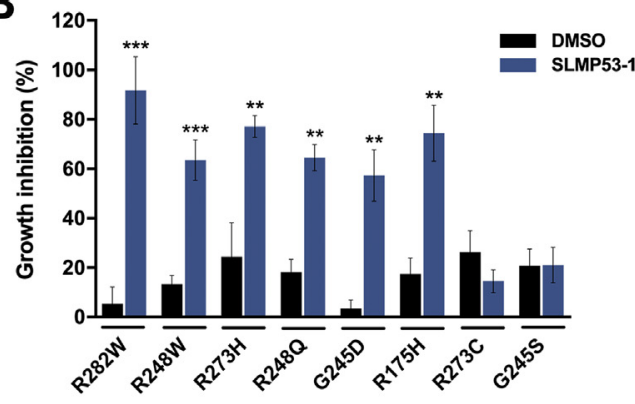

D

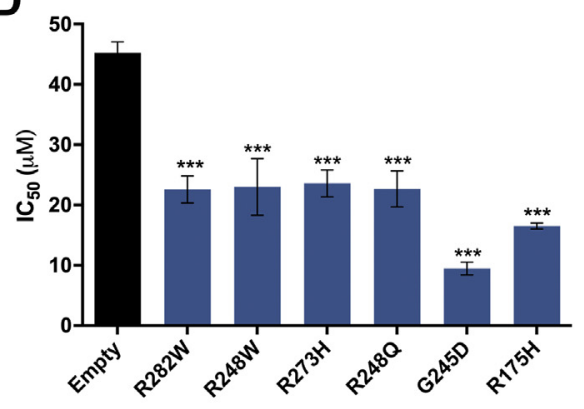

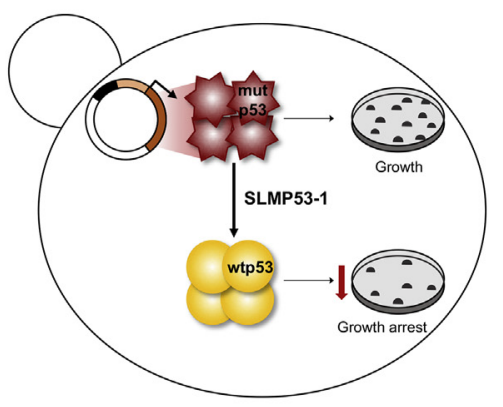

$\mathbf{E}$

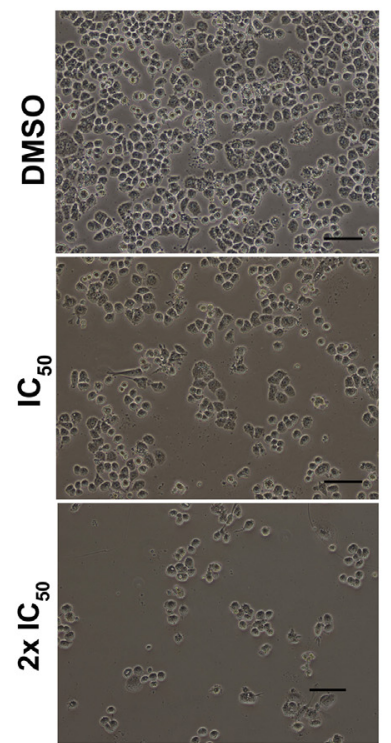

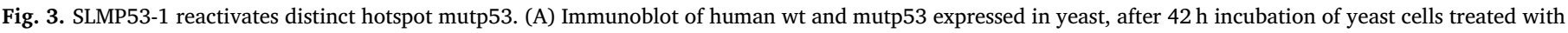

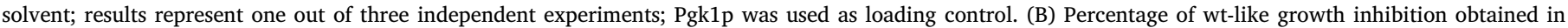

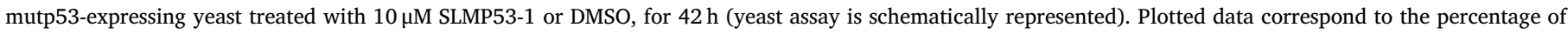

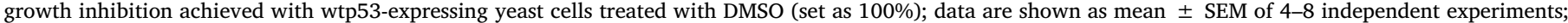

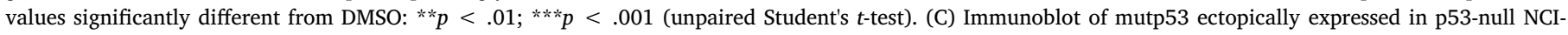

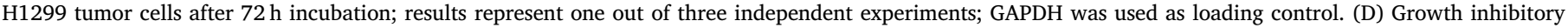

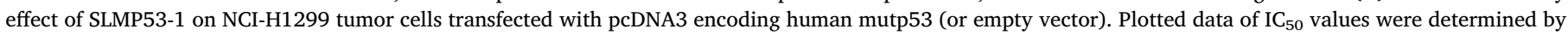

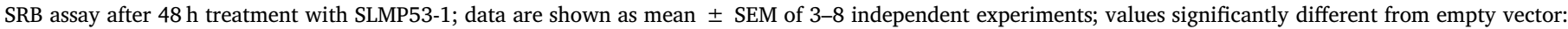

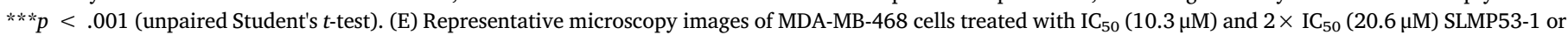

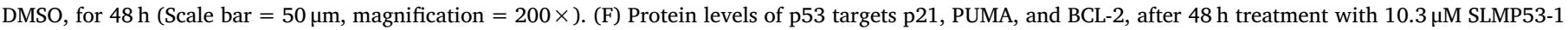
$(+)$ or DMSO $(-)$, in MDA-MB-468 cells. Immunoblots are representative of three independent experiments.

between the protein and DNA through a small molecule [44]. Furthermore, an in silico study of mutp53 R273H with curcumin, alpinetin and flavokawain has hypothesized that these small molecules might also rescue mutp53:DNA contact by binding between the protein and DNA next to the mutation site [45].

It is worth noting that this work also validates the yeast model as a reliable cell system for the high-throughput screening of mutp53 reactivators. This simplified and highly effective yeast-based assay may streamline the identification of improved mutp53 reactivators with therapeutic application in cancer treatment.

Interestingly, all mutp53 tested appear to have a similar functional activity when tested in yeast. Nevertheless, this homogeneity contrasts with the heterogeneous ability of SLMP53-1 to reactivate mutp53 occurring at the same codon but with different amino acid substitutions. Thus, it would be worth doing further studies to better understand the inability of SLMP53-1 to reactivate mutp53 Y220C, R273C, and G245S. By addressing this question, deeper insights on mutp53 pharmacology will be achieved, paving new ways in anticancer drug discovery by targeting mutp53.

\section{Conclusion}

Because of its high prevalence in human cancers and critical role in tumor formation and progression, mutp53 is one of the most promising targets in anticancer drug development [1]. As such, the search for more efficient and selective therapeutic options, capable of reactivating a broader panel of mutp53 forms, remains a priority, particularly in the context of precision medicine. The present work reinforces the clinical potential of SLMP53-1 as a p53-activating agent, with encouraging applications in personalized cancer therapy by extending the possibility of treatment to a broader panel of patients harboring both wtp53 and distinct structural or contact mutp53 forms.

\section{Acknowledgments}

This work received the financial support from the European Union (FEDER funds through Programa Operacional Factores de Competitividade - COMPETE) and National Funds (FCT/MEC, Fundação para a Ciência e Tecnologia and Ministério da Educação e Ciência) through the projects UID/QUI/50006/2019, COMPETE 2020 
(POCI-01-0145-FEDER-006684/POCI-01-0145-FEDER-007440) and the BioTecNorte operation (NORTE-01-0145-FEDER-000004), (3599PPCDT) PTDC/DTP-FTO/1981/2014 - POCI-01-0145-FEDER-016581 and UID/QUI/0081/2013; the Italian Association for Cancer Research, AIRC (IG\#5506 to G.F.), Compagnia S. Paolo, Turin, Italy (Project 2017.0526 to G.F.) and Ministry of Health, (Project $5 \times 1000,2013$ and 2015; Current research 2016). We also thank FCT for the financial support through CEECIND/01772/2017 (M.M.M. Santos), PTDC/QUIQOR/29664/2017, UID/DTP/04138/2013, IF/01272/2015 (A. Carvalho), IF/00780/2015 (F. Marcelo) and fellowships SFRH/BD/ 119144/2016 (H. Ramos), PD/BD/114046/2015 (A. S. Gomes), SFRH/ BD/128673/2017 (J. B. Loureiro), SFRH/BD/96189/2013 (S. Gomes), SFRH/BPD/110640/2015 (C. Oliveira) and PD/BI/135334/2017 (V. Barcherini), and the Programa Operacional Potencial Humano (POCH), specifically the BiotechHealth Programme (PD/00016/2012).

\section{Declaration of Competing Interest}

An international patent application protecting the compound disclosed in this manuscript has been filed by the following authors $\mathrm{S}$. Gomes, J. Soares, M.M.M. Santos, and L. Saraiva.

\section{Appendix A. Supplementary data}

Supplementary data to this article can be found online at https:// doi.org/10.1016/j.bbagen.2019.129440.

\section{References}

[1] M.J. Duffy, N.C. Synnott, J. Crown, Mutant p53 as a target for cancer treatment, Eur. J. Cancer 83 (2017) 258-265, https://doi.org/10.1016/j.ejca.2017.06.023.

[2] L. Bouaoun, et al., TP53 variations in human cancers: new lessons from the IARC TP53 database and genomics data, Hum. Mutat. 37 (9) (2016) 865-876, https:// doi.org/10.1002/humu.23035.

[3] IARC_TP53_Database. R19, August 2018. http://p53.iarc.fr/TP53SomaticMutations. aspx. ].

[4] A.C. Joerger, A.R. Fersht, Structure-function-rescue: the diverse nature of common p53 cancer mutants, Oncogene 26 (15) (2007) 2226-2242, https://doi.org/10. 1038/sj.onc. 1210291.

[5] K. Sabapathy, D.P. Lane, Therapeutic targeting of p53: all mutants are equal, but some mutants are more equal than others, Nat. Rev. Clin. Oncol. 15 (1) (2018) 13-30, https://doi.org/10.1038/nrclinonc.2017.151.

[6] V.J. Bykov, et al., Restoration of the tumor suppressor function to mutant p53 by a low-molecular-weight compound, Nat. Med. 8 (3) (2002) 282-288, https://doi.org/ 10.1038/nm0302-282.

[7] K.Y. Salim, S.M. Vareki, W.R. Danter, J. Koropatnick, COTI-2, a new anticancer drug currently under clinical investigation, targets mutant p53 and negatively modulates the PI3K/AKT/mTOR pathway, Eur. J. Cancer 69 (2016) S19.

[8] V.J.N. Bykov, et al., Targeting mutant p53 for efficient cancer therapy, Nat. Rev. Cancer 18 (2) (2018) 89-102, https://doi.org/10.1038/nrc.2017.109.

[9] Saraiva, L.S., M. Maria Manuel; Pereira, N. A. L.; Pereira, C.; Soares, J.; Gomes, S.; Leão, M.; Monteiro, A., Tryptophanol-derived oxazoloisoindolinones: small-molecule p53 activators. European patent application n ${ }^{\circ}$ EP14739561.0 (pending) and US patent $n^{\circ} 20160347765$ (granted). 2016.

[10] J. Soares, et al., Reactivation of wild-type and mutant p53 by tryptophanolderived oxazoloisoindolinone SLMP53-1, a novel anticancer small-molecule, Oncotarget 7 (4) (2016) 4326-4343, https://doi.org/10.18632/oncotarget.6775.

[11] P. Monti, et al., Dominant-negative features of mutant TP53 in germline carriers have limited impact on cancer outcomes, Mol. Cancer Res. 9 (3) (2011) 271-279, https://doi.org/10.1158/1541-7786.MCR-10-0496.

[12] A.S. Gomes, et al., The crystal structure of the R280K mutant of human p53 explains the loss of DNA binding, Int. J. Mol. Sci. 19 (4) (2018), https://doi.org/10.3390/ ijms19041184.

[13] M. Kitayner, et al., Structural basis of DNA recognition by p53 tetramers, Mol. Cell 22 (6) (2006) 741-753, https://doi.org/10.1016/j.molcel.2006.05.015.

[14] V. Hornak, et al., Comparison of multiple Amber force fields and development of improved protein backbone parameters, Proteins 65 (3) (2006) 712-725, https:// doi.org/10.1002/prot.21123.

[15] P.J.D. Stephens, F. J, C.F. Chabalowski, M.J. Frisch, Ab initio calculation of vibrational absorption and circular dichroism spectra using density functional force fields, J. Phys. Chem. 98 (1994) 11623-11627.

[16] J. Tomasi, B. Mennucci, R. Cammi, Quantum mechanical continuum solvation models, Chem. Rev. 105 (8) (2005) 2999-3093, https://doi.org/10.1021/ cr9904009.

[17] C.I.C. Bayly, W. Cornell, P.A. Kollman, A well-behaved electrostatic potential based method using charge restraints for deriving atomic charges: the RESP model, The Journal of Physical Chemistry 97 (1993) 10269-10280.

[18] Y.P. Pang, Successful molecular dynamics simulation of two zinc complexes bridged by a hydroxide in phosphotriesterase using the cationic dummy atom method, Proteins: Structure, Function, \& Genetics 45 (3) (2001) 183-189.

[19] Y.P.X. Pang, J.E. Yazal, F.G. Prendergas, Successful molecular dynamics simulation of the zinc-bound farnesyltransferase using the cationic dummy atom approach, Protein Science 9 (10) (2000) 1857-1865.

[20] G.M.G. Morris, D. S, R.S. Halliday, R. Huey, W.E. Hart, R.K. Belew, A.J. Olson, Automated docking using a Lamarckian genetic algorithm and an empirical binding free energy function, J. Comput. Chem. 19 (1998) 1639-1662.

[21] J. Wang, et al., Development and testing of a general amber force field, J. Comput. Chem. 25 (9) (2004) 1157-1174, https://doi.org/10.1002/jcc.20035.

[22] M.A.V.-H. Iglesias-Arteaga, G. A, J.M. Méndez-Stivalet, A. Galano, J.R. AlvarezIdaboy, The Baeyer-Villiger reaction of 23-oxosapogenins, ARKIVOC (2005) 109-126.

[23] R.R. Cádenas, J. Lagúnez-Otero, R. Cetina, Semiempirical studies on the transition structure of the Baeyer and Villiger rearrangement. The reaction of acetone with alkyl and aryl peracids, J. Mol. Struct. THEOCHEM 497 (2000) 211-225.

[24] J.P.C. Ryckaert, H.J.C. Berendsen, Numerical integration of the cartesian equations of motion of a system with constraints: molecular dynamics of n-alkanes, Journal of Computational Physics 23 (1977) 327-341.

[25] S.K. Miyamoto, P. A., Settle: An analytical version of the SHAKE and RATTLE algorithm for rigid water models, J. Comput. Chem. 13 (8) (1992) 952-962.

[26] T.Y. Darden, L. Pedersen, Particle mesh Ewald: An N.log(N) method for Ewald sums in large systems, J. Chem. Phys. 98 (1993) 10089-10092.

[27] D. Dourado, M. Swart, A.T.P. Carvalho, Why the Flavin Adenine Dinucleotide (FAD) cofactor needs to be covalently linked to complex II of the electron-transport chain for the conversion of FADH2 into FAD, Chemistry 24 (20) (2018) 5246-5252, https://doi.org/10.1002/chem.201704622.

[28] R.D. Gietz, R.H. Schiestl, Quick and easy yeast transformation using the LiAc/SS carrier DNA/PEG method, Nat. Protoc. 2 (1) (2007) 35-37, https://doi.org/10. 1038/nprot.2007.14.

[29] Y. Reid, et al., G.S. Sittampalam, et al. (Ed.), Authentication of Human Cell Lines by STR DNA Profiling Analysis, in Assay Guidance Manual, 2004 (Bethesda (MD)).

[30] B.X. Tan, et al., Assessing the efficacy of Mdm2/Mdm4-inhibiting stapled peptides using cellular thermal shift assays, Sci. Rep. 5 (2015) 12116, , https://doi.org/10. 1038/srep12116.

[31] J. Soares, et al., DIMP53-1: a novel small-molecule dual inhibitor of p53-MDM2/X interactions with multifunctional p53-dependent anticancer properties, Mol. Oncol. 11 (6) (2017) 612-627, https://doi.org/10.1002/1878-0261.12051.

[32] L. Raimundo, et al., Improving anticancer activity towards colon cancer cells with a new p53-activating agent, Br. J. Pharmacol. (2018), https://doi.org/10.1111/bph. 14468.

[33] J. Soares, et al., Oxazoloisoindolinones with in vitro antitumor activity selectively activate a p53-pathway through potential inhibition of the p53-MDM2 interaction, Eur. J. Pharm. Sci. 66 (2015) 138-147, https://doi.org/10.1016/j.ejps.2014.10. 006.

[34] R. Jafari, et al., The cellular thermal shift assay for evaluating drug target interactions in cells, Nat. Protoc. 9 (9) (2014) 2100-2122, https://doi.org/10.1038/ nprot.2014.138.

[35] W.A. Freed-Pastor, C. Prives, Mutant p53: one name, many proteins, Genes Dev. 26 (12) (2012) 1268-1286, https://doi.org/10.1101/gad.190678.112.

[36] P.A. Muller, K.H. Vousden, Mutant p53 in cancer: new functions and therapeutic opportunities, Cancer Cell 25 (3) (2014) 304-317, https://doi.org/10.1016/j.ccr. 2014.01.021.

[37] D. Walerych, K. Lisek, G. Del Sal, Mutant p53: one, no one, and one hundred thousand, Front. Oncol. 5 (2015) 289, https://doi.org/10.3389/fonc.2015.00289.

[38] N. Tanaka, et al., Gain-of-function mutant p53 promotes the oncogenic potential of head and neck squamous cell carcinoma cells by targeting the transcription factors FOXO3a and FOXM1, Oncogene 37 (10) (2018) 1279-1292, https://doi.org/10. 1038/s41388-017-0032-z.

[39] R.L. Weinberg, D.B. Veprintsev, A.R. Fersht, Cooperative binding of tetrameric p53 to DNA, J. Mol. Biol. 341 (5) (2004) 1145-1159, https://doi.org/10.1016/j.jmb. 2004.06.071.

[40] J.M. Lambert, et al., PRIMA-1 reactivates mutant p53 by covalent binding to the core domain, Cancer Cell 15 (5) (2009) 376-388, https://doi.org/10.1016/j.ccr. 2009.03.003.

[41] Q. Zhang, et al., APR-246 reactivates mutant p53 by targeting cysteines 124 and 277, Cell Death Dis 9 (5) (2018) 439, https://doi.org/10.1038/s41419-018-0463-7.

[42] F.M. Boeckler, et al., Targeted rescue of a destabilized mutant of p53 by an in silico screened drug, Proc. Natl. Acad. Sci. U. S. A. 105 (30) (2008) 10360-10365, https://doi.org/10.1073/pnas.0805326105.

[43] X. Liu, et al., Small molecule induced reactivation of mutant p53 in cancer cells, Nucleic Acids Res. 41 (12) (2013) 6034-6044, https://doi.org/10.1093/nar/ gkt305.

[44] J.D. Wright, S.Y. Noskov, C. Lim, Factors governing loss and rescue of DNA binding upon single and double mutations in the p53 core domain, Nucleic Acids Res. 30 (7) (2002) 1563-1574.

[45] I. Malami, et al., An in silico approach in predicting the possible mechanism involving restoration of wild-type p53 functions by small molecular weight compounds in tumor cells expressing R273H mutant p53, EXCLI J 16 (2017) 1276-1287, https://doi.org/10.17179/excli2017-299. 